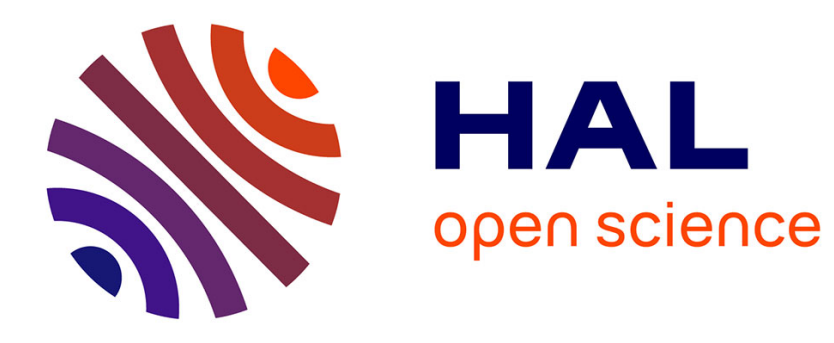

\title{
SPIN-GLASS PROPERTIES OF (Eu, Sr) S
}

\author{
H. Maletta
}

\section{To cite this version:}

H. Maletta. SPIN-GLASS PROPERTIES OF (Eu, Sr) S. Journal de Physique Colloques, 1980, 41 (C5), pp.C5-115-C5-125. 10.1051/jphyscol:1980521 . jpa-00219956

\section{HAL Id: jpa-00219956 https://hal.science/jpa-00219956}

Submitted on 1 Jan 1980

HAL is a multi-disciplinary open access archive for the deposit and dissemination of scientific research documents, whether they are published or not. The documents may come from teaching and research institutions in France or abroad, or from public or private research centers.
L'archive ouverte pluridisciplinaire HAL, est destinée au dépôt et à la diffusion de documents scientifiques de niveau recherche, publiés ou non, émanant des établissements d'enseignement et de recherche français ou étrangers, des laboratoires publics ou privés. 
SPIN-GLASS PROPERTIES OF $\left(E_{U, S r}\right) S$

\author{
H. Maletta \\ Institut für Festkörperforschung, KFA Jülich, D-5170 Jülich, RFA
}

Résumé.- Les composés isolants de la série magnétique diluée Eu $\mathrm{Sr}$. $\mathrm{S}$ montrent un

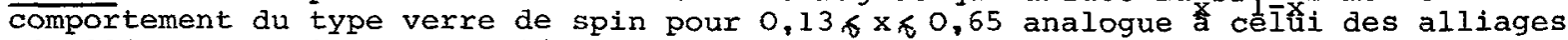
métalliques. Quelques propriétés expérimentales qui caractérisent aussi bien l'état de verre de spin que la nature de la transition sont discutées. Nous présentons une image microscopique de l'état gelé qui est basée sur la compétition de l'échange ferromagnétique entre premiers proches voisins et antiferromagnétique entre seconds proches voisins. Le modèle est supporté par l'observation de l'ordre magnétique de $\mathrm{Eu}_{\mathrm{x}} \mathrm{Sr} \mathrm{r}_{1-\mathrm{x}^{\mathrm{S}}}$ dans tout le domaine de concentration $0<\mathrm{x} \leqslant 1$, c'est-à-dire avec une trânsitexon vers 1 'état superparamagnétique pour $x<0,13$ et avec la topologie du diagramme de phase magnêtique au début du Ierromagnétisme pour $x_{C}=0,51$.

Abstract.- Insulating compounds of the magnetic dilution series Eu Sr $\mathrm{S}$ reveal spin glass behavior for $0.13 \leqslant x \leqslant 0.65$, similar to that in metallic a $110 y s$. je discuss some of the experimental properties characterizing both the spin-glass state and the nature of the transition. A microscopic picture of the "frozen" state is presented based on the competition of ferromagnetic nearest neighbor and antiferromagnetic next nearest-neighbor exchange. It is supported by the observed magnetic ordering of $\mathrm{Eu}_{x} \mathrm{Sr}_{1-x} \mathrm{~S}$ over the whole concentration range $0<\mathrm{x} \leqslant \mathrm{I}$, i.e. with the change to superparamagnetism for $x<0.13$ and the topology of the magnetic phase diagram near the onset of ferromagnetism at $x_{c}=0.51$.

1. Introduction.- Spin glasses are random magnetic systems with a puzzling magnetic ordering at low temperature. Unlike in ferromagnets and antiferromagnets, the spins "freeze" without long-range periodic spin order on cooling. The nature of both the transition into and the state of spin glass order are currently debated $/ 1,2 /$.

The "classical" spin glasses are produced by diluting magnetic ions in a nonnagnetic metallic matrix at low concentration, e.g. 1 at $\% \mathrm{Fe}$ in $\mathrm{Au}$. It is generally assumed that the long-range, oscillating RKKY interaction between the $\mathrm{Fe}$ moments mediated by the conduction electrons, together with the randomness of distances in the diluted system lead to this new type of complicated spin structure at low temperature. Numerous attemps of theoretical descriptions have been made, but nevertheless the situation is rather nuclear at present: (i) According to Edwards and Anderson (EA) /3/ the spin glass is a collective phenomenon, represented by a Heisenberg Hamiltonian with nearest neighbor exchange couplings distributed randomly according to a Gaussian. It is suggested that the frozen state then is characterized by a nonzero order parameter

$q=\left\langle\left\langle\vec{s}_{i}\right\rangle^{2}\right\rangle=\lim _{t \rightarrow \infty}\left\langle\vec{s}_{i}(0) \vec{s}_{i}(t)\right\rangle$

and in a mean-field approximation a sharp spin glass transition is found. Unfortunately, mean-field results are sometimes even nctin qualitative accord with experimental data. Other mathematical treatments of the EA-model are under discussion. (ii) Another class of explanations starts out from a picture very different from the EA-model, namely the thermal "blocking" of superparamagnetic particles. In this phenomenological theory by Tholence and Tournier /4/ the existence of isolated spin "clouds" with a distribution of moments is assumed, which can relax between different orientations. Within one cloud the spins are frozen in random directions aue to the RKKY interaction lan average number $n_{0} \approx 500$ of spins per cloud is estimated for AuFe /5/, to my knowledge, however, a definite experimental evidence for such a spin cluster structure never has been presented), whereas the different orientations of one cloud should be due to the dipolar interaction between the spins of one cloud /5/. According to Neel's model of superparamagnetism, then 
one can define an average relaxation time $\tau$ for the magnetization to jump over the potential barrier $\mathrm{E}_{\mathrm{a}}$ which separates two easy orientations of the cloud by an Arrhenius law :

$$
\tau=\tau_{0} \exp \left(E_{a} / k_{B} T\right)
$$

where $\tau_{0}$ is some intrinsic time constant. Connected with the distribution of cloud sizes and hence energies $E_{a}$, the clouds are blocked at different temperatures $\mathrm{T}_{B}$ which are determined by $\mathrm{E}_{\mathrm{a}}$ and the measuring time $\tau_{\mathrm{m}}:$

$$
\tau_{m}=\tau_{0} \exp \left(E_{a} / k_{B} T_{B}\right)
$$

In the present review emphasis is placed on characterizing typical spin-glass behavior, with a discussion in the light of the controversial concepts. We describe properties of a new spin-glass system, the insulating compounds $\mathrm{Eu}_{\mathrm{x}} \mathrm{Sr}_{1-\mathrm{x}} \mathrm{S}$, which recentIy has been investigated in detail /6/. By dilution of Eus, which orders ferromagnetically below $\mathrm{T}_{\mathrm{c}}=16.6 \mathrm{~K}$, with diamagnetic srs (with a very similar electronic structure), the magnetic ordering behavior in $\mathrm{Eu}_{\mathrm{x}} \mathrm{Sr}_{1-\mathrm{x}} \mathrm{S}$ over the whole concentration range has been studied, resulting in the magnetic phase diagram shown in figure $1 / 6,7,28 /$.

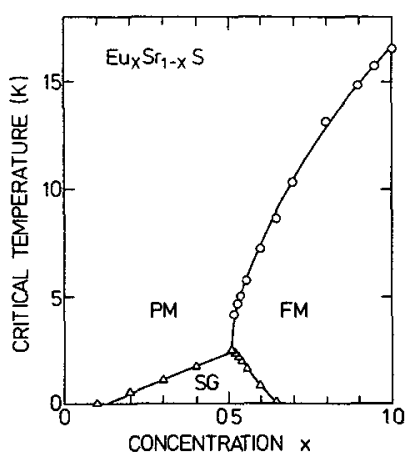

Fig. 1 Magnetic phase diagram of Eu $\mathrm{Sr} x_{1-\mathrm{S}} \mathrm{S} \cdot \mathrm{PM}=$ paramagnetism, $F M=$ ferromagnetism, ${ }^{\mathrm{SG}^{1}} \stackrel{1-\mathrm{x}}{\mathrm{spin}}$ glass $128 /$.

\section{SPIN-GLASS TEMPERATURES. - The alterna-} ting low-field susceptibility maximum has been widely used as an indicator of the characteristic temperature $T_{f}$, below which the spins are "frozen" in spin glasses.
Figure 2 shows our results on $E u_{x} S x_{1-x} S$ for Eu-concentrations $\mathrm{x}=0.10,0.25,0,30$ and 0.40 , exhibiting a nearly linear increase of the maximum position with $x$. Such a maximum in $x$ of $\mathrm{Eu}_{x} \mathrm{Sr} 1-x^{S}$ cannot be interpreted as a transition into an antiferromagnetically ordered state, since (i) the paramagnetic Curie-Weiss temperature is measured to be positive over the whole concentration regime $0<x \leqslant 1$, and (ii) neutrondiffraction spectra do not reveal magnetic Bragg lines below $\mathrm{T}_{\mathrm{f}} / 6 /$.

In adition, it is wellknown from studies of metallic spin glasses (see e.g. /8/) that the $X$-maxima are very sensitive to external magnetic fields.

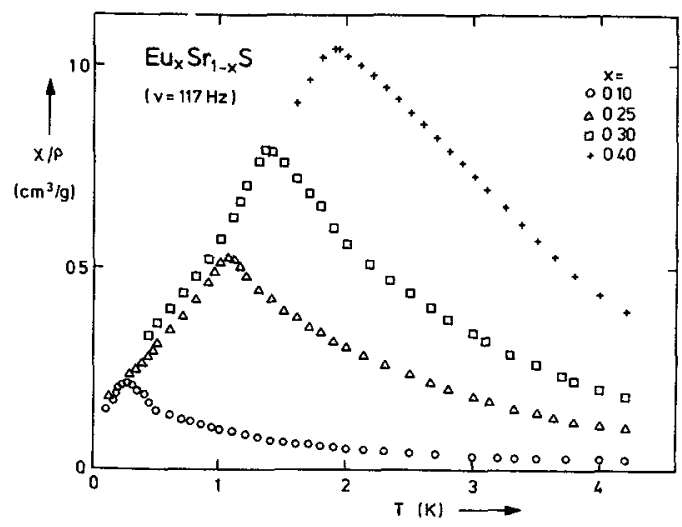

Fig. 2 Temperature dependence of the ac susceptibility of $\mathrm{Eu}_{\mathrm{x}} \mathrm{Sr}_{1-\mathrm{x}} \mathrm{S}$ with different Eu concentrations $x / 6 /$

This feature can again be observed in ( $\mathrm{Eu}, \mathrm{Sr}) \mathrm{S}$ displayed in figure 3 where the relative decrease of $X_{\text {max }}$ as function of a static magnetic field is plotted. The strong depression and rounding of $x_{\max }$ already in small fields is accompanied by a shift of $T_{f}$ to lower temperatures. As an example, a downward shift of $20 \%$ in the peak position is already obtained by applied fields of 33,20 , and $17 \mathrm{mT}$ in $\mathrm{Eu}_{\mathrm{x}} \mathrm{Sr}{ }_{1-\mathrm{x}} \mathrm{S}$ with $\mathrm{x}=0.15$, 0.25 , and 0.30 , respectively. The maximum of the susceptibility in spin glasses has attracted most theoretical activities in order to explain the transition at $\mathbb{T}_{f}$ as a thermodynamic phase transition. Under this assumption, we attempt to derive from Fig. 3 the critical exponent $\delta$ 
for the field-dependence of the EA-order parameter $q$.

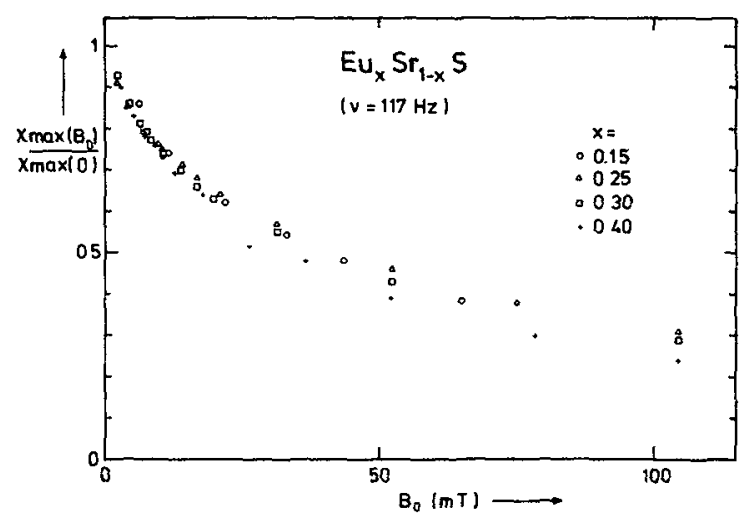

Fig. 3 Field dependence of the ac susceptibility maximum of $\mathrm{Eu} \mathrm{Sr}, \mathrm{S}, X$ ( $\mathrm{B}$ ), normalized to $B_{0}=0$, measured with superposed small static field $B_{0}$ for different $E u$ concentrations $x / 6 /$.

According to the relation obtained by Chalupa /9/

$$
q a B^{2 / \delta} \quad \text { at } T=T_{E^{\prime}}
$$

Stauffer and Binder $/ 10 /$ recently determined the exponent $\delta=4.0$ in three dimen.. sions by means of Monte Carlo simulations for the EA-Ising model. Since $q$ is related to the depression of $X_{\max }$ with $B$ by the relation

$$
q\left(T_{f}\right) \alpha 1-\chi_{\max }(B) / \chi_{\max }(0),
$$

we can compare the experimental data with the theoretical ones, as shown in figure 4, both exhibiting

$$
\delta=4.0 \text {. }
$$

of course, this good agreement between theory and experiment cannot be taken as evidence for a sharp phase transition at $T_{f}$. The experimental result of evidence for spin-glass properties in insulating $(E u, S r) S$ which is described in detail in Re $. / 6 /$,

has important consequences on the understanding of spin glasses. It demonstrates that the spin glass phenomenon is not re-stricted to metaliic alloys, contrary to the classification by Mydosh / $1 /$. Thus the long-range RKKY interaction via the conduction electrons is not to be considered as necessary condition for spin glass behavior. Regarding the interactions in ( $\mathrm{Eu}, \mathrm{Sr}) \mathrm{S}$ one has investigated intensively the coupling in pure Eus where a ferromagnetic exchange $J_{1}$ to the 12 nearest neighbors and an antiferromagnetic exchange $J_{2}$ via the anion to the 6 next-nearest neighbors are responsible for ferromagnetism in Eus with $\mathrm{J}_{2} / \mathrm{J}_{1} \approx-0.5$ (e.g. ref./11/). Hence, a basic condition creating spin-glass order is the existence of interactions of different sign, independent on the range of the interaction. We will come back to this point.
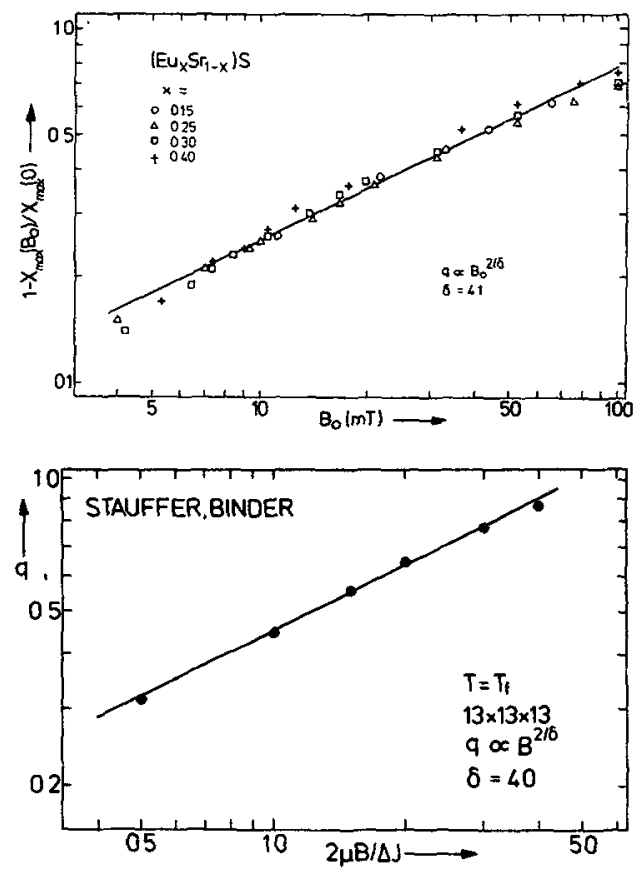

Fig. 4 Depression of $\chi_{\max }$ as function of applied

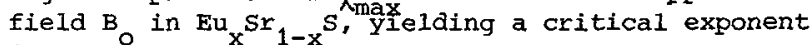
$\delta=4.1 / 6 /$. Below, the field dependence of the Edwards-Anderson order parameter $q$ at $T$ in three dimensions, calculated by Stauffer and Binder $/ 10 /$, also shows $\delta=4.0$ ( $B$ in units of $\Delta J$, the width of the interaction distribution).

3. REMANENT LIAGNETIZATION. - It is common to metallic spin glasses, that below the spin-glass temperature $T_{f}$ irreversible magnetic properties were observed. Magnetization and remanent magnetization are dependent on the thermomagnetic history and show distincly nonexponential relaxations.

In figure 5 we show some of our data from static magnetization measurements below ${ }{ }_{f}$. Both magnetization and remanent magnetization of $\mathrm{Eu}_{0.3^{S r}} 0.7^{\mathrm{S}}$ are displayed as function of 
low fields, measured after zero-field cooling and field cooling. The magnetization $\sigma(B, T)$ after zero-field cooling exhibits a smaller initial susceptibility $X_{\text {rev }}$ than $\sigma_{B}(T)$, measured after cooling in the field $B$ from $T>T_{f}$ down to $T<T_{f}$.

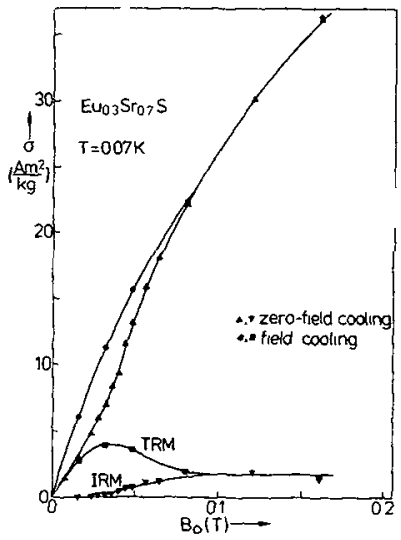

Fig. 5 Low-field magnetization of Eu $\mathrm{Sx}{ }_{7} \mathrm{~S}$ at

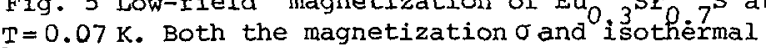
remanent magnetization (IRM), measured after zerofield cooling in increasing fiela $\bar{a}_{\text {, }}$ as well as the magnetization $\sigma_{B}$ and the thermoremanent magnetization (TRM), measured after field cooling in the field $B_{0}$ are shown $18 /$.

When the field is switched off, one observes remanent magnetizations which for low fields are smaller after zero-field cooling (IRM = isothermal remanent magnetization) than after field cooling (TRM = thermoremanent magnetization). We find the relation

$$
\sigma(B, T)=\sigma_{B}(T)-T R M(B, T)+\operatorname{IRM}(B, T),
$$

thus the $S$-shaped curvature of the virgin magnetization $\sigma(B, T)$ is directly connected to the field dependence of the remanences. Similar to metallic spin glasses one can decompose the susceptibility into a reversible and irreversible contribution

$$
\chi_{\text {rev }}(T)+\chi_{i r r}(T)=X\left(T_{f}\right) \text { for } T \leqslant T_{f}(8)
$$

where the sum stays constant below $\mathbf{T}_{\mathrm{f}}$.

$$
\begin{aligned}
& x_{\text {rev }}=\lim _{B \rightarrow 0} d \sigma(B, T) / d B, \\
& x_{\text {irr }}=\lim _{B \rightarrow 0} d \sigma(T R M) / d B .
\end{aligned}
$$

It is one of the puzzling features in spin glasses that the TRM goes through a maximum, which is even much more pronounced in (Eu, $\mathrm{Sr}$ ) $\mathrm{S}$ than for instance in metallic AuFe. In this connection, it should be noted that also in (Eu,Sr)s the typical long-time decays of the remanent magnetizations are observed (see ref. $/ 6 / 1$.

We want to emphasize on these remanent magnetizations, since the described properties are often considered as evidence for the similarity of spin glasses to simple superparamagnetism. Applying the Néel model to isolated clouds in spin glasses, in fact, the TRM is predicted to be larger than the IRM; in low fields the TRM varies as $B$, the IRM as $B^{2}$; they have the same saturated value at each temperature; and the remanent magnetization has a long-time, logarithmic decay $/ 4,5 /$. It is interesting to note here, that recently Kinzel $/ 12 /$ studied these properties by means of Monte Carlo simulations of an EAmodel with nearest neighbor interactions and obtained good qualitative agreement with all the experimental data described. Even the puzzling maximum of the TRM could be reproduced, as shown in figure 6. The maximum in the computer data is more pronounced than in AuFe, but less than in (Eu,Sr) $S$ (see Figs 5 and 6 ).
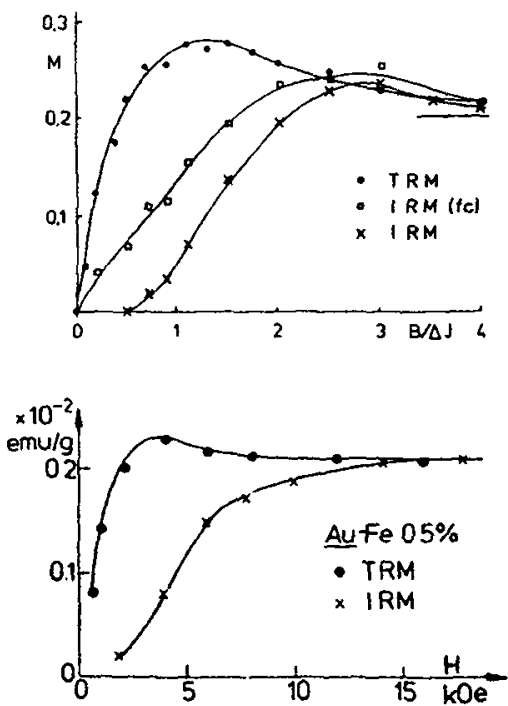

Fig. 6 Remanent magnetizations, IRM and TRM, as function of the initially applied field, obtained (below) for AuFe ( 0.5 at of $/ 4 /$ ano(above)by means of Honte Carlo calculations of an EA-Ising model /12/. IRM (fc) is calculated for a nonequilibrium start position, for details see ref./12/. 
This maximum may be explained by different field-dependences of the time-decay of TRM and IRM as found in the simulations /12/ and supported by experimental data of (Eu,sr)s shown in figures 10 and 11 of Ref. $/ 6 /$.

\section{FREQUENCY DEPENDENT SUSCEPTIBILITY MA-}

XIMUM. - As already described in sec. 1, the model of superparamagnetic clouds for spin glasses directly predicts a spin-glass temperature $z_{1}$ (or "bloking" temperature $T_{B}$ ) derendent on the sneasuring time $\tau_{m}$ (see Eg. 3).

In Eact, the maximurn of $x$ in $(\mathbb{E u}, \mathrm{Sr}) \mathrm{s}$ derived from static magnetization measurements in low fields $\left(10^{-3} \mathrm{~T}\right)$ is found to be systematically at lower temperature than $T_{f}$ determined by the ac- $\chi$ measurement on the same sample (Fig. 7).

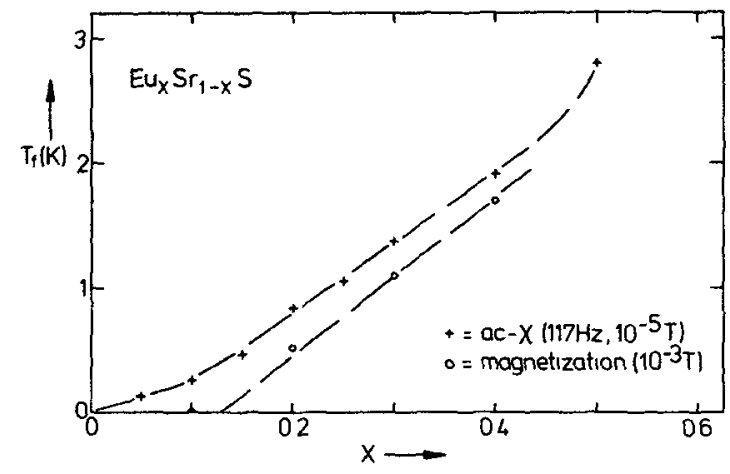

Fig. 7 Concentration dependence of the spin-glass temperature $T_{f}$ in $\mathrm{Eu} S r_{1} S$ for $\mathrm{x} \leqslant 0,5$, determined by the maximum of the susceptibility either in a low-frequency, low-field differential $X$ measurement $(117 \mathrm{~Hz}, 10-5 \mathrm{~T})$ or in a low-field, static magnetiza tion measurement $(10-3 \mathrm{~T}) / 6 /$.

By performing a series of ac- $\chi$ experiments with various measuring frequencies $\nu$, this finding is pursued and successfully supported by the observation that the position of $x_{\max }$ is shifted downward by lowering the frequency. Some of the results are compiled in Table $I$.

Appyling the concept of superparamagnetic clouds to these data, the "blocking" temperature $\mathrm{T}_{\mathrm{B}}$ should vary with the measuring frequency, following the relation :

$$
\left(T_{B}\right)^{-1}=-\frac{k_{B}}{E_{a}} \ln \left(\nu \cdot \tau_{0}\right)
$$

One realizes in figure 8 that this simple concept can formally describe the variation of $\mathrm{T}_{f}$ with frequency, measured in the ac- $x$ experiments on ( $E u, S r) S$. The fitting parameters $E_{a}$ and $\tau_{0}^{-1}$, derived from Eq. 11 and figure 8 , are found to be unreasonably high (Table I). $\tau_{0}^{-1}$ clearly cannot be interpreted as an attempt frequency, corresponding to thermal agitation from the phonon bath $\left(\sim \mathrm{k}_{B} \mathrm{~T} / \mathrm{h}\right)$, as suggested from the thermal blocking picture. In an attempt to compare the ac- and $d c-\chi$ results, the $T_{E}$ values obtained from the ac method have been extrapolated via eq. 11 to $\nu=0.02 \mathrm{~Hz}$, the measuring frequency estimated for the static (dc) method. These values $\mathrm{T}_{f}{ }^{\mathrm{ac} \phi}$, however, are still higher than the freezing temperatures $T_{f}^{d c}$ measured in the static magnetization, as shown in figure 8 and table $I$.

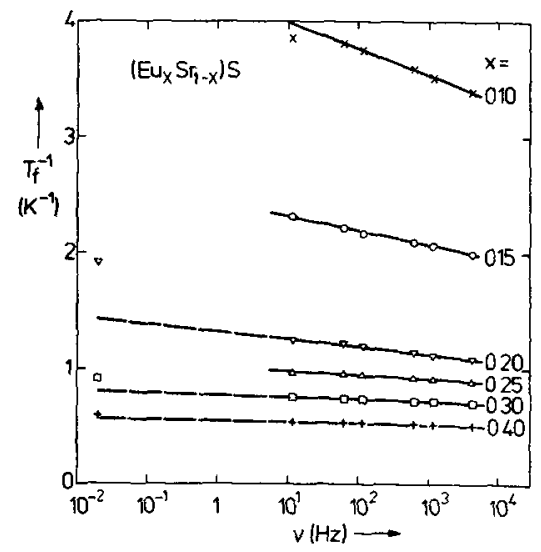

Fig. 8 Inverse temperature of the ac susceptibility maximum as function of the measuring frequency in Eu $S r$, $S$ for various Eu concentrations $x$. In addi $x_{i o n} x_{\text {the }} T_{f}$ values measured by the dc susceptibility are shown at $\nu=0.02 \mathrm{~Hz} / 6 /$.

These findings demonstrate that the cloud model of Tholence and Tournier /4/ may not be applied in this analysis. An appropriate theory for the frequency effects does not exist up to now. We suppose that the interaction between the clusters also has to be taken into account, as also suggested by Binder $/ 2 /$, since (i) the difference increases with decreasing $\mathrm{x}$ :

$$
\left(T_{f}^{\mathrm{ac} \phi}-\mathrm{T}_{\mathrm{f}}^{\mathrm{dc}}\right) \propto \mathrm{x}^{-1}
$$


and (ii) "pure" superparamagnetism with independent clusters is only observed below $\mathrm{x}=0.13$ in $\mathrm{Eu}_{\mathrm{x}} \mathrm{Sr}_{1-\mathrm{x}} \mathrm{S}$, as described in the next section.

Contrary to eq. 12 we find the difference between two $T_{f}^{a c}$ values measured at a constant frecuency interval to be inderendent 0. concentration $x$ (Table I)

$$
\Delta \mathrm{T}_{\mathrm{f}}^{\mathrm{ac}} / \Delta \ln \nu=\text { const. }
$$

Thus the discrepancy between the ac- and dc-values of $T_{f}$ seems to be different in origin from the fxequency dependence of ${ }^{1}{ }^{\prime} z$ which could be Eormal1y described by eq.11. We want to add that also the Mössbauer spectra of $\mathrm{Eu}_{\mathrm{x}} \mathrm{Sr}_{1-\mathrm{x}} \mathrm{S}$ reveal characteristic temperatures higher than $\mathrm{T}_{\mathrm{f}}^{\mathrm{ac}}$ especially for low concentrations /13/, qualitatively consistent with the above findings (since $\tau_{m} \sim^{10^{-8}}$ s.). Similar time effects were found by comparison of neutron data with $x$ data of CuMn /14/ and by comparison of ac- $\chi$ data of two (Gd,La)Al samples /15/ which also show much too high attempt frequencies $\tau_{0}{ }^{-1}$. The experimental situation in $3 d$ transition metal spin glasses, however, is not clear: while zibold /16/ concluded that the frequency effect he observed in concentrated AuFe is due to chemical clustering, Dahlberg et al./17/ did not see any frequency effect in ailute AgMn.

Anyhow the experiments on $E_{x}{ }^{S r}{ }_{1-x} S$ exhibit for the first time the time- dependent effects, shown up also near the "critical" temperature, and persisting down to very low measuring frequencies $/ 18 /$.

5. LOW-CONCENTRATION LIMIT.- In metaliic spin glasses, usually it is not possible to study the low-concentration limit of the spin-glass regime due to the continuous overlap of Kondo anomalies. In insulating $\mathrm{Eu}_{\mathrm{x}} \mathrm{Sr}_{1-\mathrm{x}} \mathrm{s}$ spin glasses, where such a restriction in tie concentration range is not present, a critical concentration $x_{p}$ for the onset of spin-glass behavior is established with

$$
x_{p} \approx 0.13
$$

by means of the $x$-dependence of the following parameters : (i) Extrapolation of

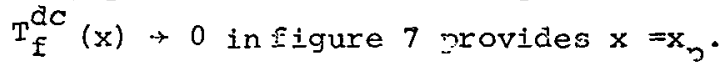
(ii) A change of the concentration depen- dence of $T_{E}^{a c}(x)$ (see Fig.7) and (iii) of the frequency dependence of $\mathrm{T}_{f}^{\mathrm{ac}}(\nu)$ (see Fig. 8 and Table I) is observed at $x=x_{p}$. Now, this experimentally determined critical concentration coincides with the percolation threshold $\mathrm{x}_{\mathrm{p}}=0.136$ calculated for a fcc lattice with nearest and next-nearest neightor interactions /19/. As pointed out in sec.2, indeed the exchange interactions in insulating ( $\mathrm{EU}, \mathrm{Sr}$ ) $\mathrm{S}$ are expected to be predominantly of short range up to the second nearest neighbors. our detailed study of $\mathrm{Eu}_{\mathrm{x}} \mathrm{Sr}{ }_{1-\mathrm{x}} \mathrm{S}$ for $\mathrm{x}<\mathrm{x}_{\mathrm{p}} / 6,20,21 /$ supports $x_{p}$ as a critical concentration, since below $x_{p}$ two striking differences in the magnetic behavior are observed in comparison to that of the spin-glass regime $\left(x>x_{p}\right)$, which is clearly demonstrated by the $x$-curves of

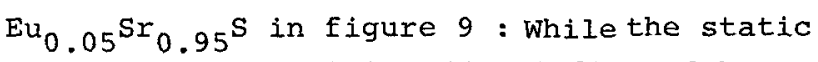
initial susceptibility (dc, indicated by $\mathrm{OHz}$ ) exhibits no maximum down to $7 \mathrm{mK}$, measured by means of a sQUID within the earth field, the real part of the dynamic susceptibility (ac) shows two distinct maxima around $\mathrm{T}_{\mathrm{a}} \approx 15 \mathrm{mK}$ and $\mathrm{T}_{\mathrm{b}} \approx 150 \mathrm{mK}$. Remember, that in the spin-glass regime $\left(x>x_{p}\right)$, both one dc-and one ac- $\chi$ maximum are found.

The strongly frequency dependent peaks near the higher temperature $\mathrm{T}_{\mathrm{b}}$ can be explained quantitatively, without adjustable parameters $/ 20 /$, by a model of small random Eu clusters. They consist of Eu atoms coupled by strong exchange forces, and experience energy barriers due to the intra-cluster dipolar energy. One particular configuration, two nearest-neighbor $\mathrm{Eu}$ atoms bonded ferromagnetically, mainly produces the anomaly near $T_{b}$. The fitting parameters $E_{a}$ and $\tau_{0}^{-1}$ show reasonable values, in contradiction to the results for $x>x_{p}$ (see sec. 4). Thus $\mathrm{T}_{\mathrm{b}}(v)$ arises from ordinary superparamagnetic behavior.

The new found maximum at the lower temperature $\mathrm{T}_{\mathrm{a}}$ is interpreted in ref. $/ 20 /$ as a result of intercluster dipolar energy, giving good agreement for the order of magnitude of $\mathrm{T}_{\mathrm{a}}$ and its concentration dependence with the calculation. If one likes to introduce the concept of a dipolar spin glass $/ 5 /$, our present understanding suggests to call $\mathrm{T}_{\mathrm{a}}$, not $\mathrm{T}_{\mathrm{b}}$ a spin-glass temperature 
(contrary to the interpretation in ref./5/.

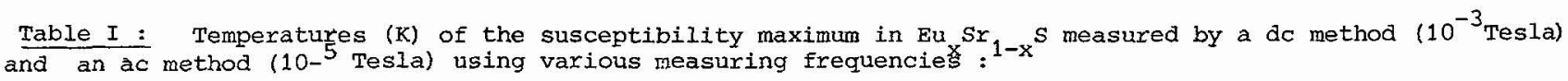

\begin{tabular}{|c|c|c|c|c|c|c|c|}
\hline$x=$ & de & $\operatorname{ac} \varnothing(0.02 \mathrm{~Hz})^{*}$ & $\mathrm{ac}(12 \mathrm{~Hz})$ & ac $(117 \mathrm{~Hz})$ & $\mathrm{ac}(4200 \mathrm{~Hz})$ & $E_{a}(K)^{* t}$ & $-\log \tau_{0}$ \\
\hline 0.10 & $<0.08$ & 0.22 & 0.260 & 0.267 & 0.295 & 9 & 13 \\
\hline 0.15 & & & 0.433 & 0.463 & 0.530 & 19 & 20 \\
\hline 0.20 & 0.52 & 0.70 & 0.807 & 0.840 & 0.920 & 36 & 21 \\
\hline 0.25 & & & 1.040 & 1.065 & 1.130 & 68 & 30 \\
\hline 0.30 & 1.10 & 1.25 & 1.350 & 1.380 & 1.450 & 111 & 37 \\
\hline 0.40 & 1.70 & 1.79 & 1.880 & 1.920 & 2.000 & 204 & 48 \\
\hline
\end{tabular}

* extrapolated values as described in the text.

* energy barriers $E_{a}(K)$ and amplitudes $\tau_{0}(\mathrm{sec})$ of Arrhenius' law applied in the analysis of $T_{f}^{a c}(V)$

The frequency dependence of $\mathrm{T}_{\mathrm{a}}$, however, again remains to be explained.

According to these results, obtained for the first time on (Eu,Sr)s, one clearly has to distinguish superparamagnetism from spinglass behavior. May be that some properties of spin glasses are successfully described by the superparamagnetic cloud model, as for instance shown in sec. 3, but other properties are not fully describable, as for instance the discussion on the frequency dependence revealed in sec. 4. A more realistic model should also take into account the interactions between the magnetic clouds in order to explain the properties of (Eu, $S r) S$ within the spin-glass regime, which has rather high concentration of magnetic atoms with many nearest neighbour $E u$ pairs and a minimum concentration for spin-glass behavior coinciding with the percolation threshold. We consider the distinction of a spin glass from superparamagnetism as an essential information provided by these experiments on $(\mathrm{Eu}, \mathrm{Sr}) \mathrm{S}$ for our understanding of spin glasses.

6. MAGNETIC CLUSTERS.- In order to further study the nature of the transition at $\mathrm{T}_{f}$, experimental results from small-angle neutron scattering and specific heat measurements on $(\mathrm{Eu}, \mathrm{Sr}) \mathrm{S}$ are presented in this section.
The use of neutron scattering techniques becomes particularly informative in a diluted magnet where magnetic inhomogeneicies are expected to be large. An estimation of the magnetic correlations in a spin-glass sample is provided directly by small-angle neutron scattering measurements. The temperature dependerce of the scattering cross-section for $\mathrm{Eu}_{0.5 \mathrm{I}^{\mathrm{Sr}}} \mathrm{r}_{.49} \mathrm{~S}$ with a relatively $\mathrm{h}_{\text {igh } \mathrm{Eu}}$ concentration near the transition to ferromagnetism at various very small scattering vectors $q\left(\mathrm{~g} \geqslant 1.52 \times 10^{-2} \AA^{-1}\right)$ is displayed in figure 10 .

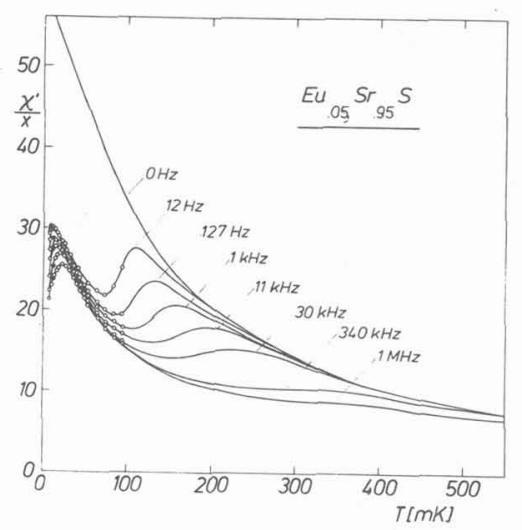

Fig. 9 Temperature dependence of the static susceptibility $(0 \mathrm{~Hz})$ and the ac susceptibility of Eu $0.05^{\mathrm{Sr}} 0.95^{\mathrm{S}}$ for various measuring frequencies $/ 20 /$. The scattering intensity has a maximum, which increases dramatically for small q-values and may be attributed to the growth of 
magnetically correlated clusters of spins on cooling. We define a magnetic cluster as a dynamical entity of a group of correlated spins which can collectively scatter a neutron just as a "paramagnetic atom". For $T \geqslant T_{f}$ the spin glass may be considered as consisting of a collection of such interacting clusters. The dramatic increase of the total scattering cross-section, observed in figure 10, indicates a predominantly ferromagnetic coupling within a cluster for this high Eu-concentration $\mathrm{x}=0.51$.

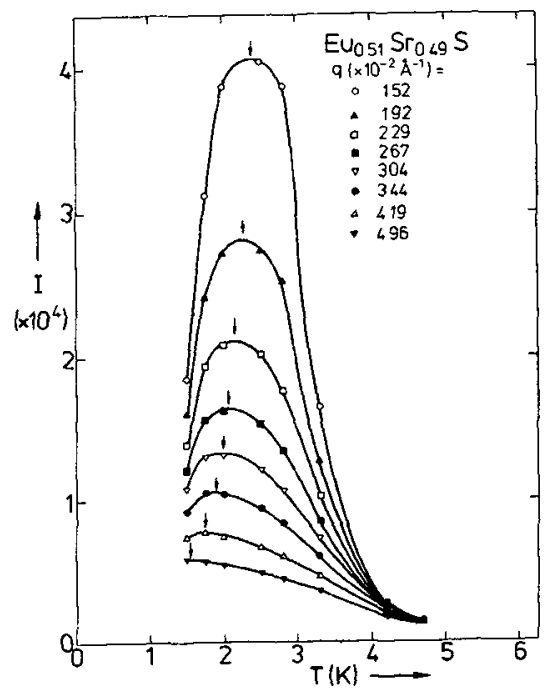

Fig. 10 Small-angle neutron scattering intensity vs. temperature for $\mathrm{Eu}_{0} \mathrm{Sr}_{4} \mathrm{~S}$ at very small scattering vectors $q$, as ${ }^{5}$ indicated. An arrow marks the temperature at the maximum for each curve.

From the q-dependence of the measured intensity, we can estimate the mean cluster size. In figure 11 the inverse intensity $\mathrm{I}^{-1}$ is plotted versus $q^{2}$, thereby straight lines are yielded. It indicates the scattering to be well represented by a Lorentzian function

$$
s(q)=s(0) /\left(1+q^{2} / k^{2}\right)
$$

implying that the magnetic correlations have the ornstein-zernike form with $K$ as the inverse correlation length

$$
\langle M(0) \quad M(x)>\alpha(I / r) \exp (-K r) \text {. }
$$

Extrapolation to $\mathrm{q}^{+} 0$ in figure 11 provides the temperature dependence of $I(0)$ and $k_{0}$, also displayed in figure 11 . One may relate these $K_{0}$ values to the root-mean-square radius of gyration for clusters using the relation

$$
\left\langle\mathrm{R}_{\mathrm{G}}^{2}\right\rangle^{1 / 2}=\sqrt{3 / \mathrm{K}} .
$$

At $T=2.5 \mathrm{~K},\left\langle\mathrm{R}_{\mathrm{G}}^{2}\right\rangle{ }^{1 / 2}$ is found to be 200

$\AA$ for $x=0.51$.

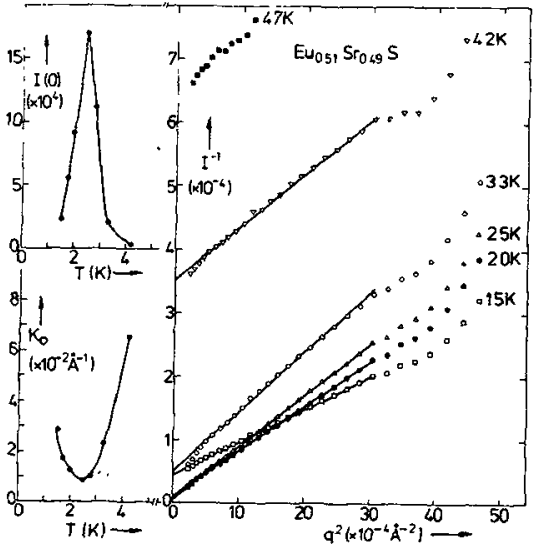

Fig. 11 Reciprocal intensity vs $\mathrm{q}^{2}$ plot for the neutron data of figure 10 at various temperatures around $\mathrm{T}_{f}$. In addition, the extrapolated $(q \rightarrow 0)$ values for intensity $I(0)$ and inverse correlation length $\mathrm{K}(0)$ are shown as function of temperature.

In figure 10 one observes the maximum position to be clearly dependent on the scattering vector $q$, with a shift toward the temperature $T_{f}$ of the low-field susceptibility maximum $X$ as $q \rightarrow 0$. Murani found a similar result on the AuFe spin glass /22/ who interpreted the behavior as evidence for $a$ continuous freezing of spin clusters, starting with thelargest cluster at the highest temperature. Soukoulis et al./23/. however, offered another interpretation of these data, thereby suggesting that the experiments cannot be maintained as firm evidence against a phase transition at $T_{f}$. They argued that the total experimental crosssection consists of two terms

$$
\frac{d \sigma}{d \Omega} \alpha T \times(q, T)+I_{B}(q, T) \text {. }
$$

The second term, called the "Bragg" term by analogy with ferromagnetism, is basically a Fourier transform of the order parameter. Their analysis suggests that a sharp phase transition is in fact reflected in $x(q, t)$. but not in the total neutron cross-section. One of the puzzling features of spin-glasses is the absence of any observably anomaly in 
the specific heat at $T_{f}$, in spite of the pronounced $x$-maximum. Recently /24/ the specific heat of the insulating spin glasses $(E u, S r) S$ was investigated, too, exhibiting the same behavior at $T_{f}$, as shown in figure 12, together with a predominant linear increase below $\mathrm{T}_{\mathrm{f}}$.

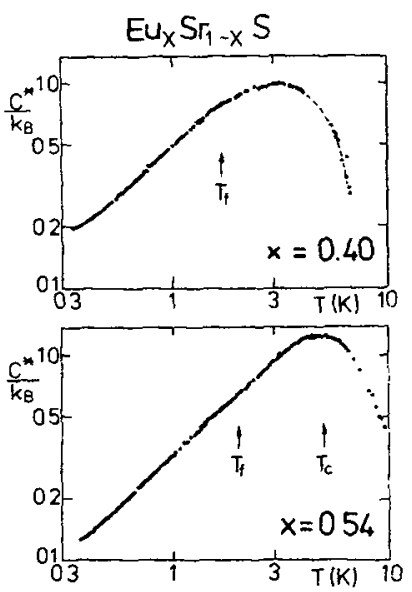

Fig. 12 Specific heat per Eu atom in a.u.as function of temperature, shown for two $\mathrm{Eu} S \mathrm{Sr} S$ samples with the transition temperatures $\mathrm{T}_{\mathrm{f}}$ and $\mathrm{T}_{\mathrm{C}}$ ) $\log$ scale) $/ 24 /$.

The different behavior of $X$ and $C$ at $T_{f}$ ' however, is not to be considered strictly as argument against the phase transition concept, if $\alpha$ (the critical exponent of the specific heat) is more negative than minus one. This seems to be fulfilled using a scaling law with the high $\delta$ (Eq. 6). Also Monte Carlo simulations of the EA-model reproduce the experimental observation of $X$ and $C$ at $T_{f}$, in contradiction to a meanfield treatment where both a cusp of $x$ and $C$ are predicted. The specific heat maximum above $T_{f}$ again reveals the importance of fairly strong magnetic correlations, developing already at $T>T_{f}$. Obviously $C$ primarily reflects the intracluster short range order, while $X$ is most sensitive to the random spin glass ordering between the clusters.

A very interesting result of figure 12 is the confirmation of a linear $\gamma . T$ term of $C(T)$ in these insulating spin glasses, which previously was detected in metallic spin glasses. It corresponds to an energy-inde- pendent density of low-lying excitations, the origin and nature of which are not yet clear. This may be considered as an interesting analogy to the dielectric and metallic glasses where also both kinds of amorphous systems show a linear dependence of $\mathrm{C}(\mathrm{T})$ at low temperature $/ 25 /$. Thus the $\gamma \cdot T$ lav of $C(T)$ appears as a universal property of disordered solids at low temperature. It may be described in terms of two-level systems in spin glasses, too, as recently suggested in ref./26/.

7. A MICROSCOPIC PICTURE. - A striking advantage of the magnetic dilution series $E_{x} u_{1-x} S$ is the possibility to study the $x$-dependence of magnetic properties over the whole concentration range $0<x \leqslant 1$, without the necessity to take into account a significant change of the exchange parameters. In addition, the short-range exchange interactions $J_{1}$ and $J_{2}$ to the nearest and nextnearest neighbors of the well-localized $4 \mathrm{f}$ spin - only moments of $\mathrm{Eu}^{2+}$ in $(\mathrm{Eu}, \mathrm{Sr}) \mathrm{S}$ are much more convenient for model calculations than the RKKY interaction and overlapping direct exchange coupling between 3d-moments in metallic spin glasses.

Several theoretical model calculations to the different magnetic ordering behavior in Eu $\mathrm{Sr}_{1-\mathrm{x}} \mathrm{S}$, shown in figure 1 , were already performed $/ 20,21,27 /$. They establish the features of the magnetic phase diagram of $\mathrm{Eu}_{\mathrm{X}} \mathrm{Sr}_{1-\mathrm{x}} \mathrm{S}$ as basic intrinsic properties of the system: (i) the percolation threshold $x_{p^{\prime}}$ (ii) the magnetic behavior for $x<x_{p^{\prime}}$ (iii) the early breakdown of ferromagnetism at $x_{c} \gg x_{p^{\prime}}$ (iv) the $x$-dependence of the Curie temperature, (v) the coexistence of long-range ferromagnetism with decoupled spins or clusters near $\mathrm{x}_{c}$. The observation mentioned in $(v)$ is discussed in ref. /28/ It is connected with a double transition $\mathrm{PM} \rightarrow \mathrm{FM}$ and $\mathrm{FM} \rightarrow \mathrm{SG}$, that can also be seen in figure 1 .

Qualitatively, one can understand these properties and the early breakdown of ferromagnetism with the appearance of the spinglass phase, far above the percolation threshold $x_{p}=0.13$ as follows (see Fig. 13), based on the competition of the ferromagnetic $\mathrm{J}_{1}$ and antiferromagnetic $\mathrm{J}_{2}$ : Near 
dilution sites there occur isolated spins (Fig. 13A) or whole clusters (Fig. 13B) aligned antiparallel to the spontaneous magnetization within the ferromagnetic phase at low temperatures.
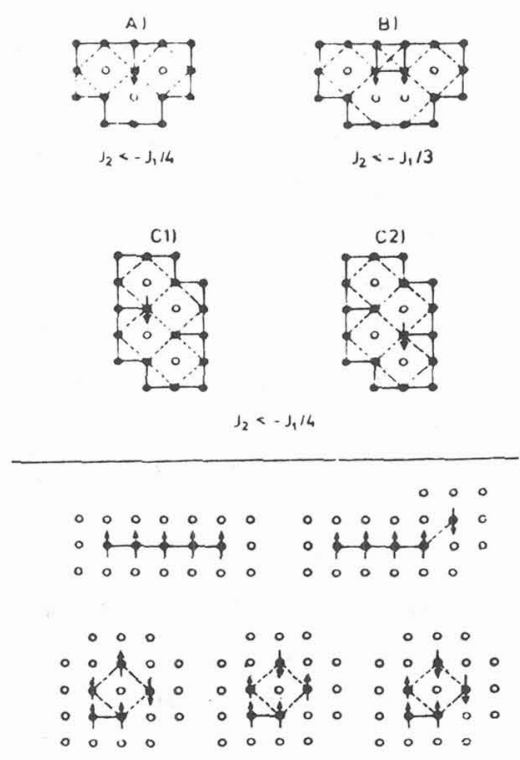

Fig. 13 Spin orientations near defect sites (empty holes) in the ground state of an Ising square with $\mathrm{J}_{1}>0$ (full lines) and $\mathrm{J}_{2}<0$ (broken lines). Spins which align antiparallel to the spontaneous magnetization are shown by arrows $/ 27 /$.

Since it is not possible to satisfy all antiferromagnetic bonds at the same time (" Erustxation"), also degenerate configurations occur with effectively decoupled clusters (Fig. 13C). The spin-glass state then is an arrangement of (in our case most1y Eerromagnetic, clusters which are coupled together with partially frustrated (and hence much weaker) 1inks. Due to frustration the ground state is expected to be highly degenerate.

8. CONCLUDING REMARKS.- The weak coupling between the clusters is considered as one of the essential features in spin glasses, leading to most of the described characteristics and probably to the long-time relaxation effects. This basic distinction between spin glass and superparamagnetism is outlined above by means of several experimental results on $(\mathrm{Eu}, \mathrm{Sr}) \mathrm{S}$. We have presented a microscopic picture of the spin- glass state, implyling magnetic clusters with weak coupling, based on the competition of exchange interactions with opposite sign. This picture is supported by experimental data of $\mathrm{Eu}_{\mathrm{x}} \mathrm{Sr}_{1-\mathrm{x}} \mathrm{S}$ together with model calculations. Today, neither experiment nor theory can convincingly answer if spinglass freezing is associated with a phase transition at $\mathrm{T}_{f} \neq 0$, or only a nonequilirium phenomenon. The most puzzling result is the anomalous slow relaxation, persisting down to time scales of minutes or hours, even near $T_{f}$ ' as revealed in the experiments on (Eu,Sr)s spin glasses.

Acknowledgements. - Sincere thanks are due to K. Binder, G. Eiselt, W. Felsch, W. Kinze1, J. Kötzler, D. Meschede, D. Stauffer, F. Steglich and W. Zinn for their collaboration on parts of the presented work. 


\section{References}

1/ Recent reviews on experiments are: Mydosh, J.A., J. Magn. Mater. 7 (1978) 237; Murani, A.P., J. Physique Colloque 39 (1978) C6-1517;

/2/ Recent theoretical reviews are; Blandin, A., J. Physique Colloq. 39 (1978) C6-1499; K. Binder in Festkörperprobleme XVII, 55 (J. TREUSCH, ed.) Vieweg, Braunschweig (1977); Anderson, P.W., in Amorphous Magnetism II, 1, Levy, $\overline{\text { R.A. }}$, Hasegawa, R., eds. (Plenum, New York) 1977 and J. Appl. Phys. 49 (1978) 1599 .

/3/ Edwards. S.F., and Anderson. P.W., J. Phys. F5 (1975) 965.

/4/ Tholence, J.L., and Tournier, R., J. Physique colloq. 35 (1974) C4-229.

/5/ Holtzberg, F,. Tholence, J.L., and Tournier, R., in Amorphous Magnetism II, (Plenum Press, New York) I 977, p. 155 .

/6/ Maletta, H., and Felsch, W., Phys. Rev. B20 (1979) 1245 .

17/ Maletta, H., and Convert, P.,Phys. Rev. lett. 42 (1979) 108.

/8/ Cannella, V., and Mydosh, J.A., Phys. Rev. B6 (1972) 4220 .

19/ Chalupa, J., solid State Commun 24 (1977) 429 .

/10/ Stauffer, D., and Binder, K., Z. Physik B30 (1978) 313.

/11/ Passe11, L., Dietrich, O.W., and AlsNielsen, J., Phys. Rev. Bl4 (1976) 4897.

/12/ Kinzel, W., J. Physique Collog. 39 (1978) C6-905, and Phys. Rev.B19 (1979)

/13/Maletta, H., and Crecelius, G., J. Physique Colloq. 37 (1976) C6-645.

/14/ Murani, A.P., and Tholence, J.I., Solid State Commun 22 (1977) 25.

/15/ Löhneysen, H.V., Tholence,J.L., and Tournier,R., J. Physique Collog. 39 (1978) C6-922.

/16/ Zibold, G., J. Phys. F8 (1978) L229.

/17/ Dahlberg, E.D., Hardiman; M., and Souletie, J., J. Phys. Lett. (Paris) 9 (1978) L389.

/18/ Maletta. H., Felsch, W., and Tholence, J.L.,J. Magn. Hagn.Mater.2(1978) 41.

/19/ Dalton, N.W., Domb, C., and Sykes, M.F, Phys. Soc., London 83 (1964) 496.

/20/ Eiselt, G., Kötzler,J., Maletta,H., Stauffer, D., and Binder, K., Phys. Rev. B19 (1979) 2664 .

/21/ Eiselt, G., Kötzler, J., Maletta, H., Binder, K., and Stauffer, D., J.Magn. Magn. Mater.13 (1979) I 46.

/22/ Murani,A.P., Phys. Rev. Lett 37 (1976) 450 .

/23/ Soukoulis, C.M., Grest,G.S., and
Levin, K., Phys. Rev. Lett, 4 I (1978) 568 .

/24/Meschede, D., Steglich, F., Felsch,W., Maletta. H.. and Zinn, W., Phys. Rev. Jett. 44 ( 1980$) 102$.

/25/Zeller, R.C., and Pohl, R.0., Phys. Rev. B4 (1971) 2029; Graebner, J.E., Golding, B., Schutz, R.J., Hsu, F.S.I., and Chen, H.S., Phys. Rev. Lett. 39 (1977) 1480 .

/26/Dasgupta, Ch., Ma, S.K., and Hu, Ch.K., Phys.Rev.B20(1979)3837.

/27/Binder, K,. Kinzel. W., Maletta, H., and Stauffer,D., IMagn. Magn. Mater. (Proceed. ICM'79, Munich).

/28/Maletta, H., J. Appl. Phys. 50 (1979)7308 (Proceed.MMM-Conf. New York, July 1979). 